\title{
Management of Hepatitis C in the Indian Context: An Update
}

\section{Prasanta K Bhattacharya* and Aakash Roy}

Department of General Medicine, North Eastern Indira Gandhi Regional Institute of Health \& Medical Sciences, Shillong, India

\begin{abstract}
Hepatitis C, a leading cause of chronic liver disease globally, is caused by Hepatitis C Virus (HCV), a hepatotropic RNA virus. $\mathrm{HCV}$ infection starts with an acute infection, mostly subclinical, which ultimately leads to chronic hepatitis in about $80 \%$ of the infected cases.

$\mathrm{HCV}$ is classified into 6 major genotypes and numerous subtypes. The global prevalence of HCV infection is about $1.6 \%$ with a majority of these infections being in adults. There is widespread heterogeneity in the prevalence of different genotypes of HCV in different geographical regions of the world. While genotype 1 is the most common worldwide, different regions of the world report variations in the prevalence of the other genotypes. Genotype 3 is the commonest genotype in India, but there is a wide variation in the distribution of the other genotypes in different parts of the country; genotype 6 , a comparatively rarer genotype, has been reported frequently from the northeast part of India.

With the introduction of new oral drugs, the Directly Acting Antivirals (DAA), the management protocols of hepatitis $\mathrm{C}$ has undergone dramatic transformation, with a paradigm shift towards an all-oral interferon-free regimen. However, developing countries like India still face a challenge with respect to accessibility and affordability of such newer regimens. Furthermore, the differences in genotypic distributions in India, with a higher prevalence of genotype 3 , which is more difficult to treat, makes the situation even more challenging. As newer antivirals are being universally used to manage $\mathrm{HCV}$ infection, economically weaker countries like India should incorporate these changes in treatment guidelines soon. However, till substantial evidence on the efficacy of the newer regimens is accrued in the Indian population, and issues on cost and accessibility are addressed, it may not yet be prudent enough to altogether discard the existing conventional modes of HCV therapy.
\end{abstract}

Keywords: Hepatitis C; HCV; Genotype; Treatment guidelines; Antivirals; Chronic hepatitis

\section{Introduction}

Hepatitis $C$ is a blood-borne viral infection that is caused by Hepatitis C Virus (HCV), a hepatotropic RNA virus, with a propensity to affect the liver. It is recognised as a major public health problem worldwide responsible for chronic liver disease and a variety of extrahepatic manifestations. It is primarily transmitted via the parenteral route which includes injection drug use, blood transfusion, unsafe injection practices, and other healthcare related procedures. $\mathrm{HCV}$ causes acute hepatitis which is mostly subclinical and gradually evolves into chronic hepatitis in about $80 \%$ of those infected [1]. For a long time hepatitis $\mathrm{C}$ remained obscure to researchers and one of the reasons is its clinically silent nature. Most patients with acute infection are symptom free and only a small proportion develops jaundice. Chronic HCV infection may be associated with vague, non-specific symptoms such as fatigue, joint pain, and discomfort in the right-upper quadrant of the abdomen. Patients usually become symptomatic when complications of chronic liver disease or extra-hepatic manifestations develop [2].

$\mathrm{HCV}$ is an enveloped positive stranded Ribonucleic Acid (RNA) virus belonging to genus Hepacivirus in the family Flaviviridae. It was first detected in 1989 using molecular biology techniques after extensive testing of serum from experimentally infected animals [3]. It is now widely recognized as one of the common aetiological agents for cirrhosis of the liver and is the leading cause of liver transplantation and the most common chronic blood borne infection in developed countries like the USA [4]. HCV is classified into 6 broad genotypes and numerous subtypes, with wide genomic variations. This has led it to be an enigmatic challenge for both clinicians and researchers.

\section{Global Epidemiology}

The total global prevalence of HCV is estimated to be at an average of $1.6 \%(1.3-2.1 \%)$, corresponding to 115 (92-149) million viraemic infections. The majority of these infections, 104 (87-124) million, are among adults (defined as $>15$ years) with an anti-HCV infection rate of $2.0 \%(1.7-2.3 \%)$. The viraemic (RNA positive) prevalence is believed to be $1.1 \%(0.9-1.4 \%)$, which corresponds to 80 (64-103) million viraemic infections [5].

Globally, genotype 1 (G1) accounts for $46 \%$ of all HCV infections among adults making it the most common, followed by G3 (22\%), G2 (13\%), G4 (13\%), G6 (2\%), and G5 (1\%) [5]. Undefined or combination of genotypes accounts for $3 \%$ of the total HCV infections. Among the subtypes, genotype $1 \mathrm{~b}$ is the most common sub-type, accounting for $22 \%$ of all infections [5]. However there are significant variations in the global distribution of genotypes. Genotype 1 is the predominantly dominating genotype in Australasia, Europe, Latin America and North America (53-71\% of all cases) [5]. Genotype 4 is the most common (71\%) in North Africa and the Middle East [5]. In Asia the predominant genotype is G3 (39\%) followed by G1 (36\%) [5].

\section{Epidemiology: The Indian Perspective}

Currently India harbours an estimated $10-15$ million chronic

*Corresponding author: Prasanta Kumar Bhattacharya, Department of Genera Medicine, North Eastern Indira Gandhi Regional Institute of Health \& Medical Sciences (NEIGRIHMS), Mawdiangdiang, Shillong 793018, India, Tel: +91 9435030138; E-mail: pkbdr78@gmail.com

Received September 10, 2015; Accepted October 13, 2015; Published October 23, 2015

Citation: Bhattacharya PK, Roy A (2015) Management of Hepatitis C in the Indian Context: An Update. J Liver 4: 187 doi:10.4172/2167-0889.1000187

Copyright: (c) 2015 Bhattacharya PK, et al. This is an open-access article distributed under the terms of the Creative Commons Attribution License, which permits unrestricted use, distribution, and reproduction in any medium, provided the original author and source are credited. 
carriers of $\mathrm{HCV}$, which is a major cause of liver related mortality and morbidity of the country [6]. The prevalence of Hepatitis C Virus (HCV) infection in the general population is estimated to be around $0.5 \%-1.5 \%$ [6]. However, the prevalence of HCV is variable in different high risk populations according to various studies and there is still a paucity of data from large multi-centric studies. Studies on voluntary or mixed donors have reported a prevalence of hepatitis $\mathrm{C}$ below $2 \%$ [4]. In a large community based systematic study from the Indian state of West Bengal the prevalence was documented to be around $0.71 \%$ [7]. On the contrary, a comparatively smaller study from the north eastern state of Arunachal Pradesh showed a much higher prevalence of hepatitis C of 7.89\% [8]. A recent study done in Mizoram, another state in the north east of the country, showed a HCV prevalence of $71.2 \%$ among the active injection drug addicts [9]. There continues to be variations in reporting the HCV prevalence, depending upon the geographical region population sub-groups included in these studies.

Overall, genotype 3 is the predominant genotype (63.85\%) followed by genotype $1(25.72 \%)$ in India [6]. However there is a genotypic difference in different geographical regions of India, with genotype 3 being the commonest in northern, eastern and western India while genotype 1 is the commonest in the southern states of India (Figure 1). Besides the widespread prevalence of genotypes 3 and 1, there is also a trend of increased prevalence of genotypes 4 and 6 in certain regions of the country. Genotype 4 is found mostly in south Indian patients from the states of Andhra Pradesh and Tamil Nadu [6]. Genotype 6 is found to be prevalent exclusively in patients from north eastern parts of the India [6]. It is also to be noted that there is also increased prevalence of genotype 6 in various parts of the neighbouring country of Myanmar which shares its boundaries with the north-eastern states of India thus showing an ecological niche for HCV genotype 6 in north-eastern India and adjacent geographical areas [10]. Genotype 2 has rarely been reported from India whereas genotype 5 is yet to be reported ${ }^{6}$. Such epidemiological studies and trend analysis are important in the wake of licensure of the newer generation DAA which may revolutionize the management of HCV. However, it must be stressed that the reported distribution of the various genotypes can be expected to change with increasing migration of population and changes in high risk behaviour and lifestyle.

\section{Virological Diagnosis of Hepatitis C}

Virological diagnosis of HCV infection is based on two categories of laboratory tests: (i) indirect tests - serologic assays detecting specific antibody to HCV (anti-HCV) and (ii) direct tests - assays that can detect, quantify, or characterize the components of HCV viral particles, such as HCV RNA and core antigen. Anti-HCV is typically identified by using Enzyme-Linked Immunosorbent Assay (ELISA) or Chemiluminescence Assays (CIA) (Table 1). Newer generation assays have made the diagnosis by these tests highly specific. Confirmation of serological reactive tests may be done by a Nucleic Acid Test (NAT) for detection of HCV RNA. A third way for diagnosis is detection of the core antigen of HCV by ELISA. Evaluations in transfusion settings have shown that the HCV core Ag assay detects HCV infection as effective as NAT [11]. A simplified approach to the diagnosis of HCV is depicted in Figure 2.

\section{Treatment Strategies of HCV}

\section{Whom to treat}

According to the current guidelines antiviral treatment is recommended for all patients with chronic HCV infection, except those with limited life expectancy due to non-hepatic causes [12]. If resources limit the ability to treat all infected patients immediately as recommended, then it is most appropriate to treat those at greatest risk of disease complications before treating those with less advanced disease or persons with risk of HCV transmission or in whom treatment may reduce transmission (Tables 2 and 3 )

\section{How to treat}

The selection of a treatment regimen will be determined by several factors. These include HCV genotype, treatment history, stage of fibrosis, medications for comorbid medical conditions, and adherence factors. The presence of associated co-morbid conditions like advancing age, chronic kidney disease, co-infection with HIV make the treatment protocols more complicated. Based on the joint recommendations issued by the American Association for the Study of Liver Diseases (AASLD) and the Infectious Diseases Society of America, in collaboration with the International Antiviral Society USA

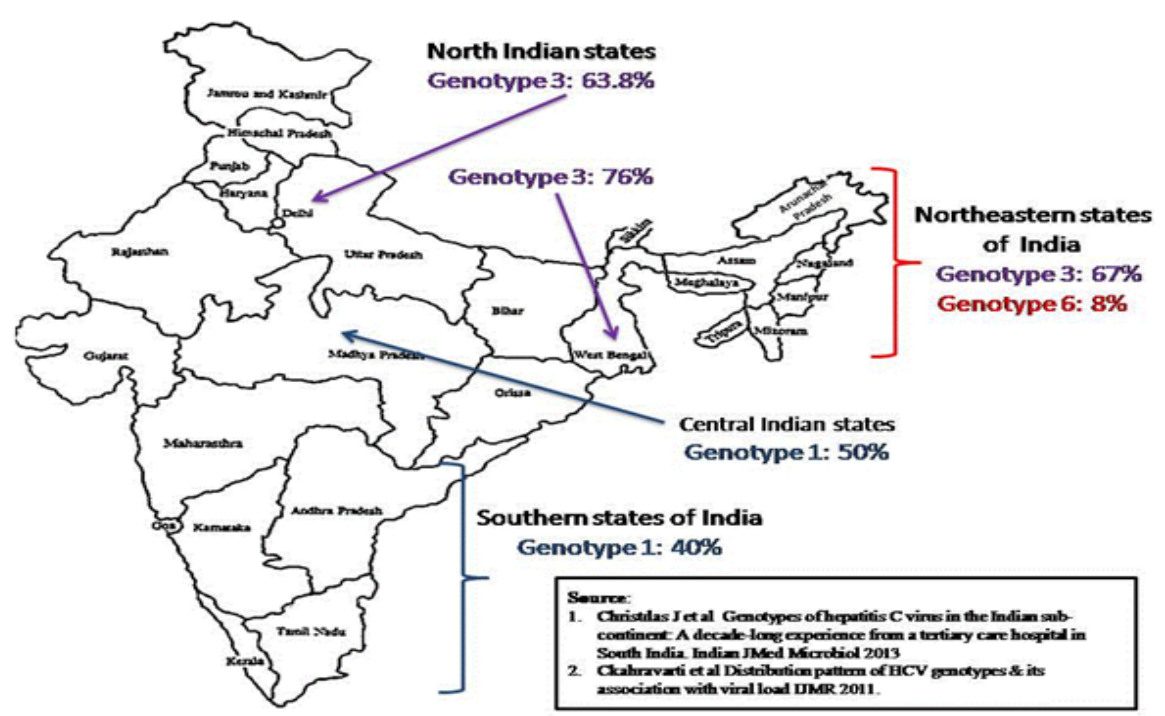

Figure 1: Showing regional distribution of HCV genotypes in India. 


\begin{tabular}{|c|c|c|c|c|}
\hline \multirow{2}{*}{$\begin{array}{c}\text { Type of ELISA test } \\
(\text { ELISA/CIA) }\end{array}$} & \multicolumn{4}{|c|}{ Antigenic targets for HCV diagnosis } \\
\cline { 2 - 5 } & Core & NS3 & NS4a & NS5b \\
\hline ELISA $1^{\text {st }}$ Generation & - & - & + & - \\
\hline ELISA $2^{\text {nd }}$ Generation & + & + & + & - \\
\hline ELISA $3^{\text {rd }}$ Generation/ CIA & + & + & + & + \\
\hline
\end{tabular}

*Modified from AASLD, IDSA, IAS-USA. Recommendations for testing, managing, and treating hepatitis $C$.

Table 1: Showing various ELISA techniques and their ability to detect different antigenic targets for HCV diagnosis*.

(IAS-USA2015), the brief summary of recent treatment guidelines for $\mathrm{HCV}$ is as follows [12].

Genotype 1: New interferon-free regimens comprising of Direct Acting Antiviral (DAA) are now the standard of care for the treatment of chronic hepatitis $C$ genotype 1 infection. For initial therapy of treatment-naive patients with genotype 1 infection, four regimens with similar efficacy are recommended [12-15] (a) ledipasvir-sofosbuvir (b) ombitasvir-paritaprevir-ritonavir with or without ribavirin, (c) sofosbuvir plus simeprevir with or without ribavirin, or (d) daclatasvir plus sofosbuvir with or without ribavirin.

For retreatment of patients with genotype 1 who had no resolution despite prior therapy with peg-interferon and ribavirin, one of following regimens that have similar efficacy are recommended [12]: (a) ledipasvir-sofosbuvir with or without ribavirin, (b) ombitasvirparitaprevir-ritonavir and dasabuvir with or without ribavirin, (c) sofosbuvir plus simeprevir with or without ribavirin, or (d) daclatasvir plus sofosbuvir with or without ribavirin. The use of ribavirin and the duration of therapy depend on the HCV genotype 1 subtype and on cirrhosis status. The duration of therapy with ledipasvir-sofosbuvir is 12 weeks without cirrhosis; those with cirrhosis can receive ledipasvirsofosbuvir for 24 weeks or ledipasvir-sofosbuvir plus ribavirin for 12 weeks.

In patients with genotype 1 infection who previously failed sofosbuvir plus ribavirin, with or without peginterferon, the recommended regimen is ledipasvir-sofosbuivr plus ribavirin [12]. The duration of therapy is 12 weeks without cirrhosis and 24 weeks with cirrhosis. For treatment-naive and treatment-experienced patients with genotype 1 infection, the new benchmark for sustained virologic response rates is $90 \%$ or greater. The major barrier to treatment with all new therapies is the extremely high cost of a treatment course.

Genotype 2: The standard of care for initial treatment of HCV genotype 2 consists of sofosbuvir plus ribavirin, which typically achieves an SVR rate of $90 \%$ or better; the duration of therapy, is 12 weeks in patients without cirrhosis and 16 weeks in those with cirrhosis [12]. For initial treatment of patients with $\mathrm{HCV}$ genotype 2 who are intolerant to ribavirin, the 12-week regimen of daclatasvir plus sofosbuvir provides a new ribavirin-free option $[16,17]$. The recommended retreatment of patients with genotype 2 and prior treatment failure with peginterferon and ribavirin consists of sofosbuvir plus ribavirin for 16 or 24 weeks; the optimal duration remains unclear [12]. For retreatment of patients with genotype 2 who previously failed therapy with sofosbuvir plus ribavirin, the recommended regimen is either (a) daclatasvir plus sofosbuvir (with or without ribavirin) for 24 weeks or (b) sofosbuvir plus ribavirin plus peginterferon for 12 weeks [12]. The demand and interest for studies that examine future therapies for genotype 2 are somewhat limited given the very high SVR rates with the all-oral regimen of sofosbuvir plus ribavirin.

Genotype 3: With new direct-acting antiviral treatments, genotype 3 has emerged as the most difficult genotype to treat. The recent FDA approval of daclatasvir has significantly expanded and improved treatment options for patients with genotype 3 infection.

Three regimens are recommended for genotype 3 treatmentnaive patients [12,18]: (1) daclatasvir plus sofosbuvir for 12 weeks (in patients without cirrhosis), (2) daclatasvir plus sofosbuvir, with or without ribavirin, for 24 weeks (in patients with cirrhosis), or (3) sofosbuvir plus ribavirin plus peginterferon for 12 weeks (with or without cirrhosis).

The recommended regimens for genotype 3 treatment-experienced patients who have failed prior treatment with peginterferon and ribavirin are [12]: (1) daclatasvir plus sofosbuvir for 12 weeks in patients without cirrhosis and 24 weeks along with ribavirin in those with cirrhosis, or (2) sofosbuvir plus ribavirin plus peginterferon for 12 weeks (with or without cirrhosis).

The recommended regimen for genotype 3 treatment-experienced patients who have failed prior treatment with sofosbuvir consists of either [12] (1) daclatasvir plus sofosbuvir plus ribavirin for 24 weeks, or

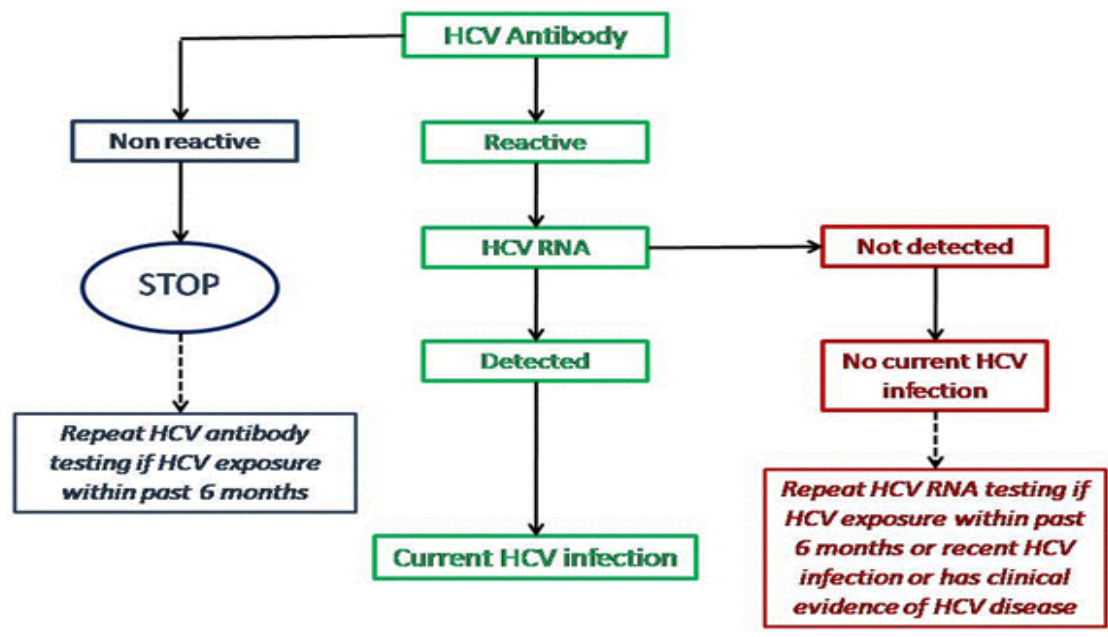

Figure 2: Algorithmic approach to HCV diagnosis. 


\begin{tabular}{|l|}
\hline $\begin{array}{l}\text { Highest priority for treatment owing to highest risk for severe } \\
\text { complications }\end{array}$ \\
\hline Advanced fibrosis or compensated cirrhosis \\
\hline Organ transplant recipients \\
\hline Type 2 or 3 cryoglobulinemia with end-organ manifestations \\
\hline Proteinuria, nephrotic syndrome, or membranoproliferative glomerulonephritis \\
\hline High priority for treatment owing to high risk for complications \\
\hline Fibrosis \\
\hline HIV-1 coinfection \\
\hline Hepatitis B virus coinfection \\
\hline Other coexistent liver disease (e.g., nonalcoholic steatohepatitis) \\
\hline Debilitating fatigue \\
\hline Type 2 diabetes mellitus (insulin-resistant) \\
\hline Porphyria cutanea tarda \\
\hline
\end{tabular}

Table 2: Hepatic complications and extra-hepatic disease conditions likely to benefit from early antiviral treatment for HCV [12].

Men who have sex with men with high-risk sexual practices

Active injection-drug users

Incarcerated persons

Persons on long-term hemodialysis

$\mathrm{HCV}$-infected women of childbearing potential wishing to get pregnant

Infected health care workers who perform exposure-prone procedures

Table 3: Persons with risk of HCV transmission where antiviral treatment may reduce transmission [12]

(2) sofosbuvir plus ribavirin plus peginterferon for 12 weeks. The major barrier to treatment and retreatment with sofosbuvir plus daclatasvir regimens in patients with genotype 3 infection remains the high cost of therapy, particularly with the 24-week all-oral combinations.

Genotype 4: For initial therapy of treatment-naive patients with genotype 4 infection, three regimens with similar efficacy are recommended $[12,19]$ in the (a) ledipasvir-sofosbuvir for 12 weeks, (b) ombitasvir-paritaprevir-ritonavir plus ribavirin for 12 weeks, or (c) sofosbuvir plus ribavirin for 24 weeks. For retreatment of patients with genotype 4 who previously failed therapy with peginterferon and ribavirin, the recommended regimens are the same as initial therapy, except they also include the option to use a 12-week course of sofosbuvir plus ribavirin plus peginterferon [12]. The investigational medications grazoprevir and elbasvir have promising activity against genotype $4 \mathrm{HCV}$ infection [12]. As in other modes of therapy the major barrier to treatment of patients with genotype 4 infection is the high cost of a treatment course.

Genotype 5 \& 6: Recommendations for initial treatment or retreatment are based on in vitro data and limited experience in clinical trials. The recommended regimen for initial treatment or retreatment of patients with genotype 5 or 6 is a 12-week course of ledipasvirsofosbuvir $[20,21]$.

The recommended regimen for retreatment of patients with genotype 5 or 6 who previously failed peginterferon plus ribavirin consists of [12] (a) ledipasvir-sofosbuvir for 12 weeks or (b) sofosbuvir plus ribavirin plus peginteferon for 12 weeks. The fixed dose combination grazoprevir-elbasvir is a promising investigational agent to treat genotype 5 or 6 infection [12].

Besides identification of genotype the treatment modalities are further sub-classified when associated with other co-morbidities like chronic kidney disease, organ transplantations and HIV co-infection. Furthermore there are certain genetic variations especially the one near the IL-28B gene have been associated with response to HCV therapy with peg-Interferon and ribavirin. Though these IL-28B gene polymorphisms may have a lesser role to play in the era of DAAs certain studies $[22,23]$ have shown that such gene polymorphisms are associated with virolological response rates in both genotype 1 and genotype 3 .

\section{Implications of Newer Treatment Modalities - Indian Scenario}

With the rapid development of newer drugs in the armamentarium against $\mathrm{HCV}$ the management of $\mathrm{HCV}$ is undergoing a global revolution. Drugs like sofosbuvir are destined to change the current treatment policies and bring about a revolution in the way we think of HCV treatment. However, in the Indian scenario, one needs to consider the genotype patterns, the stage of the disease and affordability of treatment before implementation of the proposed international guidelines.

Unlike in western countries where genotype 1 is more common, the most prevalent HCV genotype in India is genotype 3. Prior to the AASLD-IDSA-IAS-USA guidelines of 2015, most guidelines [24] had clubbed together "genotype 2 and 3" as easier to treat genotypes. However experience from India has shown that genotype 3 is a comparatively difficult genotype to treat [25]. Studies have shown that this genotype is associated with higher steatosis, has a faster progression to fibrosis [26] and has higher incidence of hepatocellular carcinoma [27] along with a poorer treatment response as compared to genotype 2. In an attempt to follow the western guidelines, although it is often presumed that the Indian population would respond in a similar way as Americans or Japanese respond, but it has been shown to be otherwise [25]. It is with this background that one must consider the consensus statement of 2014 recommended by the HCV task force of the Indian National Association for Study of the Liver on HCV infection in India [25].

These guidelines recommend standard of care therapy for chronic hepatitis $C$ patients with presently available drugs while awaiting the entry of newer DAA into the Indian market. At this point of time, when the entire western scientific community is highlighting the positive effect of the DAA, with the AASLD-2015 guidelines considering Peg-IFNa/RBV therapy as "not recommended", local compulsions in resource limited countries like India would probably still require the continued use of standard treatment regimes and would even consider use of conventional IFN. Where cost is a limiting factor in developing countries like India, relatively less efficacious but cheaper modes of therapies like INF may still be considered as an alternative option, as long as newer DAAs are unaffordable to the majority of the patients. As an example, in the neighbouring country of Pakistan, more than 20,000 persons have received therapy with conventional IFN, which has been funded by the state [28].

Another major point of discordance in the Indian guidelines, as compared to international guidelines, has been the recommendation to consider deferring treatment for certain groups of patients like those with (i) low levels of fibrosis, (ii) those with failed interferon therapy, (iii) are interferon-intolerant and (iv) stable patients after liver and kidney and liver transplant. For such patient groups it will probably be better to wait till such safer therapies become freely available and affordable in India.

However with the arrival of Sofosfubir in early 2015 the Indian guidelines are also expected to undergo a dramatic change. Although efforts are on to bring these drugs within the reach of people at an affordable cost, it is not clear as to how much time it will take. Time and more intense studies demonstrating the efficacy of the DAAs in 
the Indian patients is needed before a national consensus guideline incorporating regimens at par with international guidelines can be implemented.

\section{Conclusion}

Hepatitis C continues to be a major global threat leading to development of chronic hepatitis, cirrhosis and hepatocellular carcinoma. The distribution of HCV has wide geographic variations with multiple genotypes and subtypes. The pattern of distribution in India too is diverse with a distinct geographic heterogeneity in distributions of the different genotypes. Furthermore, an enigmatic presence of HCV genotype 6 in a certain ecologic niche in the northeastern part of the country makes the management more challenging. As the treatment undergoes dramatic changes with newer drugs revolutionizing the management protocols, India too has to incorporate these newer changes. Nevertheless, keeping in mind the accessibility and affordability of the newer regimens in a lower-middle income country like India, it is perhaps not yet time to completely abandon the conventional modes of therapy which are currently being used.

\section{References}

1. Forman MS, Valsamakis A, Versalovic J, Carrol KC, Funke G (2011) Hepatitis C virus. In: Murray's Manual of Clinical Microbiology. 10th ed. Washington: American Society of Microbiology Press 1437-1455.

2. Di Bisceglie AM (1998) Hepatitis C. The Lancet 351:351-355.

3. Choo QL, Kuo G, Weiner AJ, Overby LR, Bradley DW, et al. (1989) Isolation of a cDNA clone derived from a blood-borne non-A, non-B viral hepatitis genome. Science 244: 359-362.

4. Mukhopadhya A (2008) Hepatitis C in India. Journal of Biosciences 33: 465473.

5. Gower E, Estes C, Blach S, Razavi-Shearer K, Razavi H (2014) Global epidemiology and genotype distribution of the hepatitis $\mathrm{C}$ virus infection. Journal of hepatology 61: 45-57.

6. Christdas J, Sivakumar J, David J, Daniel H, Raghuraman S, et al. (2013) Genotypes of hepatitis $C$ virus in the Indian subcontinent: A decade long experience from a tertiary care hospital in South India. Indian J Med Microbiol 31: $349-353$

7. Chowdhury A, Santra A, Chaudhuri S, Dhali GK, Chaudhuri S, et al. (2003) Hepatitis $C$ virus infection in the general population: a community-based study in West Bengal, India. Hepatology 37: 802-809.

8. Phukan AC, Das HK, Mahanta J (2001) HCV activity in an isolated community in north east India. Indian J Pathol Microbiol 44: 403-405.

9. Chelleng PK, Borkakoty BJ, Chetia M, Das HK, Mahanta J (2008) Risk of hepatitis $\mathrm{C}$ infection among injection drug users in Mizoram, India. Indian J Med Res 128: 640-646.

10. Wasitthankasem R, Vongpunsawad S, Siripon N, Suya C, Chulothok P, et al. (2015) Genotypic Distribution of Hepatitis C Virus in Thailand and Southeast Asia. PLoS ONE 10: e0126764.

11. Seme K, Poljak M, Babic DZ, Mocilnik T, Vince A (2005) The role of core antigen detection in management of hepatitis C: A critical review. J Clin Virol 32: 92-101.

12. https://www.iasusa.org/content/aas/didsaias $\% E 2 \% 80 \% 93$ usa-hcv-guidancerecommendations-testing-managing-and-treating-hepatitis-c
13. Kowdley KV, Gordon SC, Reddy KR, Rossaro L, Bernstein DE, et al. (2014) Ledipasvir and sofosbuvir for 8 or 12 weeks for chronic HCV without cirrhosis. N Engl J Med 370: 1879-1888.

14. Ferenci P, Bernstein D, Lalezari J, Cohen D, Luo Y, et al. (2014) ABT-450/rombitasvir an dasabuvir with or without ribavirin forHCV. N Engl J Med 370: 1983-1992.

15. Lawitz E, Poordad FF, Pang PS, Hyland RH, Ding X, et al. (2013) Sofosbuvir and ledipasvir fixed-dose combination with and withoutribavirin in treatmentnaive and previously treated patients with genotype 1 hepatitis $C$ virus infection (LONESTAR): an open-label, randomised, phase 2 trials. Lancet 383: 515-523.

16. Lawitz E, Mangia A, Wyles D, Rodriguez-Torres M, Hassanein T, et al. (2013) Sofosbuvir for previously untreated chronic hepatitis $C$ infection. N Engl J Med 368: 1878-1887.

17. Jacobson IM, Gordon SC, Kowdley KV, Yoshida EM, Rodriguez-Torres M, et al. (2013) Sofosbuvir for hepatitis C genotype 2 or 3 in patients without treatment options. N Engl J Med 368: 1867-1877.

18. Zeuzem S, Dusheiko GM, Salupere R, Mangia A, Flisiak R, et al. (2013) Sofosbuvir1ribavirin for 12 or 24 weeks for patients with HCV genotype 2 or 3: the VALENCE trial [Abstract]. Proceedings of the $64^{\text {th }}$ Annual Meeting of the American Association for the Study of Liver Diseases (AASLD) Washington, DC Abstract 1085.

19. Kapoor R, Kohli A, Sidharthan S, Sims Z, Petersen TL, et al. (2014) All oral treatment for genotype 4 chronic hepatitis $C$ infection with sofosbuvir and ledipasvir: interim results from the NIAID SYNERGY trial [Abstract] Proceedings of the 65th Annual Meeting of the American Association for the Study of Liver Diseases (AASLD) Boston, MA. Abstract 240.

20. Abergel A, Loustaud-Ratti V, Metivier S, Jiang D, Kersey K, et al. (2015) Ledipasvir/sofosbuvir for the treatment of patients with chronic genotype 4 or $5 \mathrm{HCV}$ infection [Abstract]. Proceedings of the $50^{\text {th }}$ Annual Meeting of the European Association for the Study of the Liver (EASL) Vienna, Austria.

21. Gane EJ, Hyland RH, An D, Svarovskaia ES, Pang PS, et al. (2014) High efficacy of LDV/SOF regimens for 12 weeks for patients with HCV genotype 3 or 6 infection [Abstract]. Proceedings of the 65th Annual Meeting of the American Association for the Study of Liver Diseases (AASLD) Boston, MA.

22. Chinnaswamy S (2014) Genetic variants at the IFNL3 locus and their association with hepatitis $C$ virus infections reveal novel insights into host-virus interactions. J Interferon Cytokine Res 34: 479-497.

23. Chinnaswamy S, Das K, Bairagya BB, Bhattacharyya C, Shalimar, et al. (2014) Association of IL28B single nucleotide polymorphism rs8099917 with response totreatment in genotype $3 \mathrm{HCV}$-infected patients from India. Trop Gastroenterol 35: 96-102.

24. http://www.hcvguidelines.org/sites/default/files/full_report.pdf

25. Puri P, Anand AC, Saraswat VA, Acharya SK, Dhiman RK, et al. (2014) Consensus Statement of HCV Task Force of the Indian National Association for Study of the Liver (INASL). Part I: Status Report of HCV Infection in India. Journal of clinical and experimental hepatology 4: 106-116.

26. Bochud PY, Cai T, Overbeck K, Bochud M, Dufour JF, et al. (2009) Genotype 3 is associated with accelerated fibrosis progression in chronic hepatitis $\mathrm{C}$. J Hepatol 51: 655-666.

27. Nkontchou G, Ziol M, Aout M (2011) HCV genotype 3 is associated with a higher hepatocellular carcinoma incidence in patients with ongoing viral $\mathrm{C}$ cirrhosis. J Viral Hepat 18: 516-522.

28. Akbar H, Idrees M, Manzoor S (2009) Hepatitis $\mathrm{C}$ virus infection: a review of the current and future aspects and concerns in Pakistan. J Gen Mol Virol 1: 12-18. 